\title{
Iterative algorithms for monotone inclusion problems, fixed point problems and minimization problems
}

Jong Soo Jung*

"Correspondence: jungjs@dau.ac.kr;
jungjs@mail.donga.ac.kr
Department of Mathematics,
Dong-A University, Busan, 604-714,
Korea
Korea

\begin{abstract}
We introduce new implicit and explicit iterative schemes for finding a common element of the set of fixed points of $k$-strictly pseudocontractive mapping and the set of zeros of the sum of two monotone operators in a Hilbert space. Then we establish strong convergence of the sequences generated by the proposed schemes to a common point of two sets, which is a solution of a certain variational inequality. Further, we find the unique solution of the quadratic minimization problem, where the constraint is the common set of two sets mentioned above. As applications, we consider iterative schemes for the Hartmann-Stampacchia variational inequality problem and the equilibrium problem coupled with fixed point problem.
\end{abstract}

MSC: 47H05; 47H09; 47H10; 47J05; 47J07; 47J25; 47J20; 49M05

Keywords: maximal monotone operator; inverse-strongly monotone operator; strictly pseudocontractive mapping; fixed points; variational inequality; zeros; minimum norm problem

\section{Introduction}

Let $H$ be a real Hilbert space with the inner product $\langle\cdot, \cdot\rangle$ and the induced norm $\|\cdot\|$. Let $C$ be a nonempty closed convex subset of $H$, and let $T: C \rightarrow C$ be a self-mapping on $C$. We denote by $F(T)$ the set of fixed points of $T$, that is, $F(T):=\{x \in C: T x=x\}$.

Let $A: C \rightarrow H$ be a single-valued nonlinear mapping, and let $B: H \rightarrow 2^{H}$ be a multivalued mapping. Then we consider the monotone inclusion problem (MIP) of finding $x \in H$ such that

$$
0 \in A x+B x
$$

The set of solutions of the MIP (1.1) is denoted by $(A+B)^{-1} 0$. That is, $(A+B)^{-1} 0$ is the set of zeros of $A+B$. The MIP (1.1) provides a convenient framework for studying a number of problems arising in structural analysis, mechanics, economics and others; see, for instance $[1,2]$. Also, various types of inclusion problems have been extended and generalized, and there are many algorithms for solving variational inclusions. For more details, see [3-5] and the references therein.

The class of pseudocontractive mappings is one of the most important classes of mappings among nonlinear mappings. We recall that a mapping $T: C \rightarrow H$ is said to be

@2013 Jung; licensee Springer. This is an Open Access article distributed under the terms of the Creative Commons Attribution License (http://creativecommons.org/licenses/by/2.0), which permits unrestricted use, distribution, and reproduction in any medium, provided the original work is properly cited. 
$k$-strictly pseudocontractive if there exists a constant $k \in[0,1)$ such that

$$
\|T x-T y\|^{2} \leq\|x-y\|^{2}+k\|(I-T) x-(I-T) y\|^{2}, \quad \forall x, y \in C .
$$

Note that the class of $k$-strictly pseudocontractive mappings includes the class of nonexpansive mappings as a subclass. That is, $T$ is nonexpansive (i.e., $\|T x-T y\| \leq\|x-y\|$, $\forall x, y \in C$ ) if and only if $T$ is 0 -strictly pseudocontractive. The mapping $T$ is also said to be pseudocontractive if $k=1$, and $T$ is said to be strongly pseudocontractive if there exists a constant $\lambda \in(0,1)$ such that $T-\lambda I$ is pseudocontractive. Clearly, the class of $k$-strictly pseudocontractive mappings falls into the one between classes of nonexpansive mappings and pseudocontractive mappings. Also, we remark that the class of strongly pseudocontractive mappings is independent of the class of $k$-strictly pseudocontractive mappings (see [6]). Recently, many authors have been devoting the studies on the problems of finding fixed points for pseudocontractive mappings (see, for example, [7-10] and the references therein).

Recently, in order to study the MIP (1.1) coupled with the fixed point problem, many authors have introduced some iterative schemes for finding a common element of the set of solutions of the MIP (1.1) and the set of fixed points of a countable family of nonexpansive mappings (see $[4,5,11]$ and the references therein).

Inspired and motivated by the above-mentioned recent works, in this paper, we introduce new implicit and explicit iterative schemes for finding a common element of the set of the solutions of the MIP (1.1) with a set-valued maximal monotone operator $B$ and an inverse-strongly monotone mapping $A$ and the set of fixed points of a $k$-strictly pseudocontractive mapping $T$. Then we establish results of the strong convergence of the sequences generated by the proposed schemes to a common point of two sets, which is a solution of a certain variational inequality. As a direct consequence, we find the unique solution of the quadratic minimization problem:

$$
\|\tilde{x}\|^{2}=\min \left\{\|x\|^{2}: x \in F(T) \cap(A+B)^{-1} 0\right\} .
$$

Moreover, as applications, we consider iterative algorithms for the Hartmann-Stampacchia variational inequality problem and the equilibrium problem coupled with fixed point problem of nonexpansive mappings.

\section{Preliminaries and lemmas}

Let $H$ be a real Hilbert space, and let $C$ be a nonempty closed convex subset of $H$. In the following, we write $x_{n} \rightarrow x$ to indicate that the sequence $\left\{x_{n}\right\}$ converges weakly to $x$. $x_{n} \rightarrow x$ implies that $\left\{x_{n}\right\}$ converges strongly to $x$.

Recall that mapping $f: C \rightarrow C$ is said to be contractive if there exists $l \in[0,1)$ such that

$$
\|f(x)-f(y)\| \leq l\|x-y\|, \quad \forall x, y \in C
$$

A mapping $A$ of $C$ into $H$ is called inverse-strongly monotone if there exists a positive real number $\alpha$ such that

$$
\langle x-y, A x-A y\rangle \geq \alpha\|A x-A y\|^{2}
$$


for all $x, y \in C$. For such a case, $A$ is called $\alpha$-inverse-strongly monotone. If $A$ is an $\alpha$-inverse-strongly monotone mapping of $C$ into $H$, then it is obvious that $A$ is $\frac{1}{\alpha}$ Lipschitzian and continuous. Let $B$ be a mapping of $H$ into $2^{H}$. The effective domain of $B$ is denoted by $\operatorname{dom}(B)$, that is, $\operatorname{dom}(B)=\{x \in H: B x \neq \emptyset\}$. A multi-valued mapping $B$ is said to be a monotone operator on $H$ if $\langle x-y, u-v\rangle \geq 0$ for all $x, y \in \operatorname{dom}(B), u \in B x$, and $v \in B y$. A monotone operator $B$ on $H$ is said to be maximal if its graph is not properly contained in the graph of any other monotone operator on $H$. For a maximal monotone operator $B$ on $H$ and $r>0$, we may define a single-valued operator $J_{r}=(I+r B)^{-1}: H \rightarrow \operatorname{dom}(B)$, which is called the resolvent of $B$ for $r$. Let $B$ be a maximal monotone operator on $H$, and let $B^{-1} 0=\{x \in H: 0 \in B x\}$. It is well known that $B^{-1} 0=F\left(J_{r}\right)$ for all $r>0$ and the resolvent $J_{r}$ is firmly nonexpansive, i.e.,

$$
\left\|J_{r} x-J_{r} y\right\|^{2} \leq\left\langle x-y, J_{r} x-J_{r} y\right\rangle, \quad \forall x, y \in H,
$$

and that the resolvent identity

$$
J_{\lambda} x=J_{\mu}\left(\frac{\mu}{\lambda} x+\left(1-\frac{\mu}{\lambda}\right) J_{\lambda} x\right)
$$

holds for all $\lambda, \mu>0$ and $x \in H$. It is worth mentioning that the resolvent operator $J_{\lambda}$ is nonexpansive and 1-inverse-strongly monotone, and that a solution of the MIP (1.1) is a fixed point of the operator $J_{\lambda}(I-\lambda A)$ for all $\lambda>0$ (see [11]).

In a real Hilbert space $H$, we have

$$
\|x-y\|^{2}=\|x\|^{2}+\|y\|^{2}-2\langle x, y\rangle
$$

for all $x, y \in H$ and $\lambda \in \mathbb{R}$. For every point $x \in H$, there exists a unique nearest point in $C$, denoted by $P_{C} x$, such that

$$
\left\|x-P_{C} x\right\|=\inf \{\|x-y\|: y \in C\} .
$$

$P_{C}$ is called the metric projection of $H$ onto $C$. It is well known that $P_{C}$ is nonexpansive, and $P_{C}$ is characterized by the property

$$
u=P_{C} x \quad \Longleftrightarrow \quad\langle x-u, u-y\rangle \geq 0, \quad \forall x \in H, y \in C .
$$

It is also well known that $H$ satisfies the Opial condition, that is, for any sequence $\left\{x_{n}\right\}$ with $x_{n} \rightarrow x$, the inequality

$$
\liminf _{n \rightarrow \infty}\left\|x_{n}-x\right\|<\liminf _{n \rightarrow \infty}\left\|x_{n}-y\right\|
$$

holds for every $y \in H$ with $y \neq x$. For these facts, see [12].

We need the following lemmas for the proof of our main results.

Lemma 2.1 In a real Hilbert space $H$, the following inequality holds:

$$
\|x+y\|^{2} \leq\|x\|^{2}+2\langle y, x+y\rangle, \quad \forall x, y \in H .
$$


Lemma 2.2 [12] For all $x, y, z \in H$ and $\alpha, \beta, \gamma \in[0,1]$ with $\alpha+\beta+\gamma=1$, the following equality holds:

$$
\|\alpha x+\beta y+\gamma z\|^{2}=\alpha\|x\|^{2}+\beta\|y\|^{2}+\gamma\|z\|^{2}-\alpha \beta\|x-y\|^{2}-\beta \gamma\|y-z\|^{2}-\gamma \alpha\|z-x\|^{2} .
$$

Lemma 2.3 [13] Let $H$ be a Hilbert space, let $C$ be a closed convex subset of $H$. If $T$ is a $k$-strictly pseudocontractive mapping on $C$, then the fixed point set $F(T)$ is closed convex, so that the projection $P_{F(T)}$ is well defined, and $F\left(P_{C} T\right)=F(T)$.

Lemma 2.4 [13] Let $H$ be a real Hilbert space, let $C$ be a closed convex subset of $H$, and let $T: C \rightarrow H$ be a k-strictly pseudocontractive mapping. Define a mapping $T: C \rightarrow H$ by $S x=\lambda x+(1-\lambda) T x$ for all $x \in C$. Then, as $\lambda \in[k, 1), S$ is a nonexpansive mapping such that $F(S)=F(T)$.

Lemma 2.5 [14] Let C be a nonempty closed convex subset of a real Hilbert space H. Let the mapping $A: C \rightarrow H$ be $\alpha$-inverse strongly monotone, and let $r>0$ be a constant. Then we have

$$
\|(I-r A) x-(I-r A) y\|^{2} \leq\|x-y\|^{2}+r(r-2 \alpha)\|A x-A y\|^{2}, \quad \forall x, y \in C .
$$

In particular, if $0 \leq r \leq 2 \alpha$, then $I-r A$ is nonexpansive.

Lemma 2.6 [15] Let $B: H \rightarrow 2^{H}$ be a maximal monotone operator, and let $A: H \rightarrow H$ be a Lipschitz continuous mapping. Then the mapping $B+A: H \rightarrow 2^{H}$ is a maximal monotone operator.

Remark 2.1 Lemma 2.6 implies that $(A+B)^{-1} 0$ is closed and convex if $B: H \rightarrow 2^{H}$ is a maximal monotone operator and $A: H \rightarrow H$ is an inverse-strongly monotone mapping.

The following lemma is a variant of a Minty lemma (see [16]).

Lemma 2.7 Let $C$ be a nonempty closed convex subset of a real Hilbert space H. Assume that the mapping $G: C \rightarrow H$ is monotone and weakly continuous along segments, that is, $G(x+t y) \rightarrow G(x)$ weakly as $t \rightarrow 0$. Then the variational inequality

$$
\tilde{x} \in C, \quad\langle G \tilde{x}, p-\tilde{x}\rangle \geq 0, \quad \forall p \in C,
$$

is equivalent to the dual variational inequality

$$
\tilde{x} \in C, \quad\langle G p, p-\tilde{x}\rangle \geq 0, \quad \forall p \in C .
$$

Lemma 2.8 [17] Let $\left\{x_{n}\right\}$ and $\left\{z_{n}\right\}$ be bounded sequences in a real Banach space E, and let $\left\{\gamma_{n}\right\}$ be a sequence in $[0,1]$, which satisfies the following condition:

$$
0<\liminf _{n \rightarrow \infty} \gamma_{n} \leq \limsup _{n \rightarrow \infty} \gamma_{n}<1
$$


Suppose that $x_{n+1}=\gamma_{n} x_{n}+\left(1-\gamma_{n}\right) z_{n}$ for all $n \geq 1$, and

$$
\limsup _{n \rightarrow \infty}\left(\left\|z_{n+1}-z_{n}\right\|-\left\|x_{n+1}-x_{n}\right\|\right) \leq 0
$$

Then $\lim _{n \rightarrow \infty}\left\|z_{n}-x_{n}\right\|=0$.

Lemma 2.9 [18] Let $\left\{s_{n}\right\}$ be a sequence of non-negative real numbers satisfying

$$
s_{n+1} \leq\left(1-\xi_{n}\right) s_{n}+\xi_{n} \delta_{n}, \quad \forall n \geq 1,
$$

where $\{\xi\}$ and $\left\{\delta_{n}\right\}$ satisfy the following conditions:

(i) $\left\{\xi_{n}\right\} \subset[0,1]$ and $\sum_{n=1}^{\infty} \xi_{n}=\infty$;

(ii) $\limsup _{n \rightarrow \infty} \delta_{n} \leq 0$ or $\sum_{n=1}^{\infty} \xi_{n} \delta_{n}<\infty$.

Then $\lim _{n \rightarrow \infty} s_{n}=0$.

\section{Iterative schemes}

Throughout the rest of this paper, we always assume as follows: Let $H$ be a real Hilbert space, and let $C$ be a nonempty closed convex subset of $H$. Let $A: C \rightarrow H$ be an $\alpha$-inverse strongly monotone mapping, and let $B$ be a maximal monotone operator on $H$ such that the domain of $B$ is included in $C$. Let $J_{\lambda}=(I+\lambda B)^{-1}$ be the resolvent of $B$ for $\lambda>0$. Let $f$ : $C \rightarrow C$ be a contractive mapping with constant $l \in(0,1)$, and let $T: C \rightarrow C$ be a $k$-strictly pseudocontractive mapping. Define a mapping $S: C \rightarrow C$ by $S x=\lambda x+(1-\lambda) T x, \forall x \in C$, where $\lambda \in[k, 1)$. Then, by Lemma $2.4, S$ is nonexpansive.

In this section, we introduce the following iterative scheme that generates a net $\left\{x_{t}\right\}$ in an implicit way:

$$
x_{t}=t f\left(x_{t}\right)+(1-t) S J_{\lambda_{t}}\left(x_{t}-\lambda_{t} A x_{t}\right), \quad t \in(0,1)
$$

where $0<a \leq \lambda_{t} \leq b<2 \alpha$. We prove strong convergence of $\left\{x_{t}\right\}$, as $t \rightarrow 0$, to a point $\tilde{x}$ in $F(T) \cap(A+B)^{-1} 0$, which is a solution of the following variational inequality:

$$
\langle(I-f) \tilde{x}, p-\tilde{x}\rangle \geq 0, \quad \forall p \in F(T) \cap(A+B)^{-1} 0 .
$$

Equivalently, $\tilde{x}=P_{F(T) \cap(A+B)^{-1} 0}(2 I-f) \tilde{x}$.

If we take $f \equiv 0$ in (3.1), then we have

$$
x_{t}=(1-t) S J_{\lambda_{t}}\left(x_{t}-\lambda_{t} A x_{t}\right), \quad t \in(0,1) .
$$

We also propose the following iterative scheme which generates a sequence $\left\{x_{n}\right\}$ in an explicit way:

$$
x_{n+1}=\alpha_{n} f\left(x_{n}\right)+\beta_{n} x_{n}+\left(1-\alpha_{n}-\beta_{n}\right) S J_{\lambda_{n}}\left(x_{n}-\lambda_{n} A x_{n}\right), \quad \forall n \geq 0,
$$

where $\left\{\alpha_{n}\right\},\left\{\beta_{n}\right\} \subset(0,1),\left\{\lambda_{n}\right\} \subset(0,2 \alpha)$ and $x_{0} \in C$ is an arbitrary initial guess, and establish the strong convergence of this sequence to a fixed point $\tilde{x}$ of $T$, which is also a solution of the variational inequality (3.2). If we take $f \equiv 0$ in (3.4), then we have

$$
x_{n+1}=\beta_{n} x_{n}+\left(1-\alpha_{n}-\beta_{n}\right) S J_{\lambda_{n}}\left(x_{n}-\lambda_{n} A x_{n}\right), \quad \forall n \geq 0 .
$$




\subsection{Strong convergence of the implicit algorithm}

For $t \in(0,1)$, consider the following mapping $Q_{t}$ on $C$ defined by

$$
Q_{t} x=t f(x)+(1-t) S J_{\lambda_{t}}\left(x-\lambda_{t} A x\right), \quad \forall x \in C .
$$

By Lemma 2.5, we have

$$
\begin{aligned}
& \left\|Q_{t} x-Q_{t} y\right\| \\
& \quad=\left\|t f(x)+(1-t) S J_{\lambda_{t}}\left(x-\lambda_{t} A x\right)-\left(t f(y)+(1-t) S J_{\lambda_{t}}\left(y-\lambda_{t} A y\right)\right)\right\| \\
& \quad \leq t\|f(x)-f(y)\|+(1-t)\left\|S J_{\lambda_{t}}\left(x-\lambda_{t} A x\right)-S J_{\lambda_{t}}\left(y-\lambda_{t} A y\right)\right\| \\
& \quad \leq t l\|x-y\|+(1-t)\left\|\left(I-\lambda_{t} A\right) x-\left(I-\lambda_{t} A\right) y\right\| \\
& \quad \leq t l\|x-y\|+(1-t)\|x-y\| \\
& \quad=[1-(1-l) t]\|x-y\| .
\end{aligned}
$$

Since $0<1-(1-l) t<1, Q_{t}$ is a contractive mapping. Therefore, by the Banach contraction principle, $Q_{t}$ has a unique fixed point $x_{t} \in C$, which uniquely solves the fixed point equation

$$
x_{t}=t f\left(x_{t}\right)+(1-t) S J_{\lambda_{t}}\left(x_{t}-\lambda_{t} A x_{t}\right), \quad t \in(0,1) .
$$

Now, we prove strong convergence of the sequence $\left\{x_{t}\right\}$, and show the existence of $\tilde{x} \in$ $F(T) \cap(A+B)^{-1} 0$, which solves the variational inequality (3.2).

Theorem 3.1 Suppose that $F(T) \cap(A+B)^{-1} 0$. Then the net $\left\{x_{t}\right\}$ defined by the implicit method (3.1) converges strongly, as $t \rightarrow 0$, to a point $\tilde{x} \in F(T) \cap(A+B)^{-1} 0$, which is the unique solution of the variational inequality (3.2).

Proof First, we can show easily the uniqueness of a solution of the variational inequality (3.2). In fact, if $\tilde{x} \in F(T) \cap(A+B)^{-1} 0$ and $\hat{x} \in F(T) \cap(A+B)^{-1} 0$ both are solutions to (3.2). Then we have

$$
\begin{aligned}
& \langle(I-f) \tilde{x}, \hat{x}-\tilde{x}\rangle \geq 0 \\
& \langle(I-f) \hat{x}, \tilde{x}-\hat{x}\rangle \geq 0 .
\end{aligned}
$$

Adding up (3.6) and (3.7) yields

$$
\langle(I-f) \tilde{x}-(I-f) \hat{x}, \tilde{x}-\hat{x}\rangle \leq 0 .
$$

This implies that $(1-l)\|\tilde{x}-\hat{x}\|^{2} \leq 0$. So $\tilde{x}=\hat{x}$, and the uniqueness is proved. Below, we use $\tilde{x} \in F(T) \cap(A+B)^{-1} 0$ to denote the unique solution of the variational inequality (3.2).

Now, we prove that $\left\{x_{t}\right\}$ is bounded. Set $y_{t}=J_{\lambda_{t}}\left(x_{t}-\lambda_{t} A x_{t}\right)$ for all $t \in(0,1)$. Take $p \in F(T) \cap(A+B)^{-1} 0$. It is clear that $p=J_{\lambda_{t}}\left(p-\lambda_{t} A p\right)=S J_{\lambda_{t}}\left(p-\lambda_{t} A p\right)$ and $p=S p$ (by Lemma 2.4). Since $J_{\lambda_{t}}$ is nonexpansive and $A$ is $\alpha$-inverse-strongly monotone, we have 
from Lemma 2.5 that

$$
\begin{aligned}
\left\|y_{t}-p\right\|^{2} & =\left\|J_{\lambda_{t}}\left(x_{t}-\lambda_{t} A x_{t}\right)-J_{\lambda_{t}}\left(p-\lambda_{t} A p\right)\right\|^{2} \\
& \leq\left\|x_{t}-\lambda_{t} A x_{t}-\left(p-\lambda_{t} A p\right)\right\|^{2} \\
& \leq\left\|x_{t}-p\right\|^{2}+\lambda_{t}\left(\lambda_{t}-2 \alpha\right)\left\|A x_{t}-A p\right\|^{2} \\
& \leq\left\|x_{t}-p\right\|^{2} .
\end{aligned}
$$

So, we have that

$$
\left\|y_{t}-p\right\| \leq\left\|x_{t}-p\right\|
$$

Moreover, from (3.1), it follows that

$$
\begin{aligned}
\left\|x_{t}-p\right\| & =\left\|t f\left(x_{t}\right)+(I-t) S J_{\lambda_{t}}\left(x_{t}-\lambda_{t} A x_{t}\right)-p\right\| \\
& \leq\left\|t\left(f\left(x_{t}\right)-f(p)\right)\right\|+t\|f(p)-p\|+(1-t)\left\|J_{\lambda_{t}}\left(x_{t}-\lambda_{t} A x_{t}\right)-p\right\| \\
& \leq t l\left\|x_{t}-p\right\|+t\|f(p)-p\|+(1-t)\left\|y_{t}-p\right\| \\
& \leq t l\left\|x_{t}-p\right\|+t\|f(p)-p\|+(1-t)\left\|x_{t}-p\right\| \\
& \leq[1-t(1-l)]\left\|x_{t}-p\right\|+t\|f(p)-p\|,
\end{aligned}
$$

that is,

$$
\left\|x_{t}-p\right\| \leq \frac{\|f(p)-p\|}{1-l}
$$

Hence, $\left\{x_{t}\right\}$ is bounded, and so are $\left\{y_{t}\right\},\left\{f\left(x_{t}\right)\right\},\left\{A x_{t}\right\}$ and $\left\{S y_{t}\right\}$.

From (3.8) and (3.10), we have

$$
\begin{aligned}
(1-t l)^{2}\left\|x_{t}-p\right\|^{2} \leq & {\left[(1-t)\left\|y_{t}-p\right\|+t\|f(p)-p\|\right]^{2} } \\
= & (1-t)^{2}\left\|y_{t}-p\right\|^{2}+t^{2}\|f(p)-p\|^{2} \\
& \quad+2(1-t) t\|f(p)-p\|\left\|y_{t}-p\right\| \\
\leq & \left\|y_{t}-p\right\|^{2}+t M_{1} \\
\leq & \left\|x_{t}-p\right\|^{2}+\lambda_{t}\left(\lambda_{t}-2 \alpha\right)\left\|A x_{t}-A p\right\|^{2}+t M_{1},
\end{aligned}
$$

where $M_{1}>0$ is an appropriate constant. This means that

$$
\begin{aligned}
a(2 \alpha-b)\left\|A x_{t}-A p\right\|^{2} & \leq \lambda_{t}\left(2 \alpha-\lambda_{t}\right)\left\|A x_{t}-A p\right\|^{2} \\
& \leq t\left(2 l-t l^{2}\right)\left\|x_{t}-p\right\|^{2}+t M_{1} \rightarrow 0 \quad \text { as } t \rightarrow 0
\end{aligned}
$$

Since $a(2 \alpha-b)>0$, we deduce that

$$
\lim _{t \rightarrow 0}\left\|A x_{t}-A p\right\|=0 .
$$


From (2.1) and (2.3), we also obtain

$$
\begin{aligned}
\| y_{t}- & p \|^{2} \\
= & \left\|J_{\lambda_{t}}\left(x_{t}-\lambda_{t} A x_{t}\right)-J_{\lambda_{t}}\left(p-\lambda_{t} A p\right)\right\|^{2} \\
\leq & \left\langle\left(x_{t}-\lambda_{t} A x_{t}\right)-\left(p-\lambda_{t} A p\right), y_{t}-p\right\rangle \\
= & \frac{1}{2}\left(\left\|\left(x_{t}-\lambda_{t} A x_{t}\right)-\left(p-\lambda_{t} A p\right)\right\|^{2}+\left\|y_{t}-p\right\|^{2}\right. \\
& \left.-\left\|\left(x_{t}-p\right)-\lambda_{t}\left(A x_{t}-A p\right)-\left(y_{t}-p\right)\right\|^{2}\right) \\
\leq & \frac{1}{2}\left(\left\|x_{t}-p\right\|^{2}+\left\|y_{t}-p\right\|^{2}-\left\|\left(x_{t}-y_{t}\right)-\lambda_{t}\left(A x_{t}-A p\right)\right\|^{2}\right) \\
= & \frac{1}{2}\left(\left\|x_{t}-p\right\|^{2}+\left\|y_{t}-p\right\|^{2}-\left\|x_{t}-y_{t}\right\|^{2}+2 \lambda_{t}\left\langle x_{t}-y_{t}, A x_{t}-A p\right\rangle-\lambda_{t}^{2}\left\|A x_{t}-A p\right\|^{2}\right) .
\end{aligned}
$$

So, we get

$$
\begin{aligned}
\left\|y_{t}-p\right\|^{2} \leq & \left\|x_{t}-p\right\|^{2}-\left\|x_{t}-y_{t}\right\|^{2} \\
& +2 \lambda_{t}\left\langle x_{t}-y_{t}, A x_{t}-A p\right\rangle-\lambda_{t}^{2}\left\|A x_{t}-A p\right\|^{2} .
\end{aligned}
$$

Since $\|\cdot\|^{2}$ is a convex function, by (3.13), we have

$$
\begin{aligned}
\left\|x_{t}-p\right\|^{2}= & \left\|t\left(f\left(x_{t}\right)-p\right)+(1-t)\left(S J_{\lambda_{t}}\left(x_{t}-\lambda_{t} A x_{t}\right)-p\right)\right\|^{2} \\
\leq & t\left(\left\|f\left(x_{t}\right)-f(p)\right\|+\|f(p)-p\|\right)^{2}+(1-t)\left\|S y_{t}-S p\right\|^{2} \\
\leq & t\left(l\left\|x_{t}-p\right\|+\|f(p)-p\|\right)^{2}+(1-t)\left\|y_{t}-p\right\|^{2} \\
\leq & t\left(l\left\|x_{t}-p\right\|+\|f(p)-p\|\right)^{2} \\
& +(1-t)\left(\left\|x_{t}-p\right\|^{2}-\left\|x_{t}-y_{t}\right\|^{2}+2 \lambda_{t}\left\langle x_{t}-y_{t}, A x_{t}-A p\right\rangle\right) .
\end{aligned}
$$

We deduce from (3.14) that

$$
(1-t)\left\|x_{t}-y_{t}\right\|^{2} \leq\left(t+\left\|A x_{t}-A p\right\|\right) M_{2},
$$

where $M_{2}>0$ is an appropriate constant. Since $t \rightarrow 0$ and $\left\|A x_{t}-A p\right\| \rightarrow 0$, we have

$$
\lim _{t \rightarrow \infty}\left\|x_{t}-y_{t}\right\|=0
$$

Observing that

$$
\begin{aligned}
\left\|S y_{t}-x_{t}\right\| & =\left\|S y_{t}-\left(t f\left(x_{t}\right)+(1-t) S y_{t}\right)\right\| \\
& =t\left\|S y_{t}-f\left(x_{t}\right)\right\| \rightarrow 0 \quad \text { as } t \rightarrow 0,
\end{aligned}
$$

by (3.16), we obtain

$$
\begin{aligned}
\left\|S x_{t}-x_{t}\right\| & \leq\left\|S x_{t}-S y_{t}\right\|+\left\|S y_{t}-x_{t}\right\| \\
& \leq\left\|x_{t}-y_{t}\right\|+\left\|S y_{t}-x_{t}\right\| \rightarrow 0 \quad \text { as } t \rightarrow 0 .
\end{aligned}
$$


Let $\left\{t_{n}\right\} \subset(0,1)$ be a sequence such that $t_{n} \rightarrow 0$ as $n \rightarrow \infty$. Put $x_{n}:=x_{t_{n}}, y_{n}:=y_{t_{n}}$ and $\lambda_{n}:=\lambda_{t_{n}}$. Since $\left\{x_{n}\right\}$ is bounded, there exists a subsequence $\left\{x_{n_{i}}\right\}$ of $\left\{x_{n}\right\}$, which converges weakly to $\tilde{x}$.

Next, we show that $\tilde{x} \in F(T) \cap(A+B)^{-1} 0$. Since $C$ is closed and convex, $C$ is weakly closed. So, we have $\tilde{x} \in C$. Let us show $\tilde{x} \in F(T)$. Assume that $\tilde{x} \notin F(T)(=F(S))$. Since $x_{n_{i}}-\tilde{x}$ and $\tilde{x} \neq S \tilde{x}$, it follows from the Opial condition and (3.17) that

$$
\begin{aligned}
\liminf _{i \rightarrow \infty}\left\|x_{n_{i}}-\tilde{x}\right\| & <\liminf _{i \rightarrow \infty}\left\|x_{n_{i}}-S \tilde{x}\right\| \\
& \leq \liminf _{i \rightarrow \infty}\left(\left\|x_{n_{i}}-S x_{n_{i}}\right\|+\left\|S x_{n_{i}}-S \tilde{x}\right\|\right) \\
& \leq \liminf _{i \rightarrow \infty}\left\|x_{n_{i}}-\tilde{x}\right\|,
\end{aligned}
$$

which is a contradiction. So we get $\tilde{x} \in F(T)$.

We shall show that $\tilde{x} \in(A+B)^{-1} 0$. Since $\left\|x_{t}-y_{t}\right\| \rightarrow 0$ as $t \rightarrow 0$, it follows that $\left\{y_{n_{i}}\right\}$ converges weakly to $\tilde{x}$. We choose a subsequence $\left\{\lambda_{n_{i}}\right\}$ of $\left\{\lambda_{n}\right\}$ such that $\lambda_{n_{i}} \rightarrow \lambda$. Let $v \in$ $B u$. Noting that

$$
y_{t}=J_{\lambda_{t}}\left(x_{t}-\lambda_{t} A x_{t}\right)=\left(I+\lambda_{t} B\right)^{-1}\left(x_{t}-\lambda_{t} A x_{t}\right),
$$

we have that

$$
x_{t}-\lambda_{t} A x_{t} \in y_{t}+\lambda_{t} B y_{t},
$$

and so,

$$
\frac{x_{t}-y_{t}}{\lambda_{t}}-A x_{t} \in B y_{t}
$$

Since $B$ is monotone, we have for $(u, v) \in B$,

$$
\left\langle\frac{x_{t}-y_{t}}{\lambda_{t}}-A x_{t}-v, y_{t}-u\right\rangle \geq 0
$$

Since $\left\langle x_{t}-\tilde{x}, A x_{t}-A \tilde{x}\right\rangle \geq \alpha\left\|A x_{t}-A \tilde{x}\right\|^{2}$ and $x_{n_{i}}-\tilde{x}$, we have $A x_{n_{i}} \rightarrow A \tilde{x}$. Then by (3.16) and (3.18), we obtain

$$
\langle-A \tilde{x}-v, \tilde{x}-u\rangle \geq 0 .
$$

Since $B$ is maximal monotone, this implies that $-A \tilde{x} \in B \tilde{x}$, that is, $0 \in(A+B) \tilde{x}$. Hence, we have $\tilde{x} \in(A+B)^{-1} 0$. Thus, we conclude that $\tilde{x} \in F(T) \cap(A+B)^{-1} 0$.

On the one hand, we note that for $p \in F(T) \cap(A+B)^{-1} 0$,

$$
x_{t}-p=t\left(f\left(x_{t}\right)-f(p)\right)+t(f(p)-p)+(1-t)\left(S J_{\lambda_{t}}\left(x_{t}-\lambda_{t} A x_{t}\right)-p\right) .
$$

Then it follows that

$$
\begin{aligned}
\left\|x_{t}-p\right\|^{2} & =\left\langle x_{t}-p, x_{t}-p\right\rangle \\
& =\left\langle t\left(f\left(x_{t}\right)-f(p)\right), x_{t}-p\right\rangle+t\left\langle f(p)-p, x_{t}-p\right\rangle
\end{aligned}
$$




$$
\begin{aligned}
& +(1-t)\left\langle S J_{\lambda_{t}}\left(x_{t}-\lambda_{t} A x_{t}\right)-p, x_{t}-p\right\rangle \\
\leq & t l\left\|x_{t}-p\right\|^{2}+t\left\langle f(p)-p, x_{t}-p\right\rangle+(1-t)\left\|x_{t}-p\right\|^{2} \\
= & (1-(1-l) t)\left\|x_{t}-p\right\|^{2}+t\left\langle f(p)-p, x_{t}-p\right\rangle .
\end{aligned}
$$

Hence, we have

$$
\left\|x_{t}-p\right\|^{2} \leq \frac{1}{1-l}\left\langle f(p)-p, x_{t}-p\right\rangle
$$

In particular,

$$
\left\|x_{n_{i}}-p\right\|^{2} \leq \frac{1}{1-l}\left\langle f(p)-p, x_{n_{i}}-p\right\rangle
$$

Since $\tilde{x} \in F(T) \cap(A+B)^{-1} 0$, by (3.20), we obtain

$$
\left\|x_{n_{i}}-\tilde{x}\right\| \frac{1}{1-l}\left\langle f(\tilde{x})-\tilde{x}, x_{n_{i}}-\tilde{x}\right\rangle .
$$

Since $x_{n_{i}} \rightarrow \tilde{x}$, it follows from (3.21) that $x_{n_{i}} \rightarrow \tilde{x}$ as $i \rightarrow \infty$.

Now, we return to (3.20) and take the limit as $i \rightarrow \infty$ to get

$$
\|\tilde{x}-p\|^{2} \leq \frac{1}{1-l}\langle(I-f) p, p-\tilde{x}\rangle .
$$

In particular, $\tilde{x}$ solves the following variational inequality

$$
\tilde{x} \in F(T) \cap(A+B)^{-1} 0, \quad\langle(I-f) p, p-\tilde{x}\rangle \geq 0, \quad p \in F(T) \cap(A+B)^{-1} 0,
$$

or the equivalent dual variational inequality (see Lemma 2.7)

$$
\tilde{x} \in F(T) \cap(A+B)^{-1} 0, \quad\langle(I-f) \tilde{x}, p-\tilde{x}\rangle \geq 0, \quad p \in F(T) \cap(A+B)^{-1} 0 .
$$

Finally, we show that the net $\left\{x_{t}\right\}$ converges strongly, as $t \rightarrow 0$, to $\tilde{x}$. To this end, let $\left\{s_{k}\right\} \subset(0,1)$ be another sequence such that $s_{k} \rightarrow 0$ as $k \rightarrow \infty$. Put $x_{k}:=x_{s_{k}}, y_{k}:=y_{s_{k}}$ and $\lambda_{k}:=\lambda_{s_{k}}$. Let $\left\{x_{k_{j}}\right\}$ be a subsequence of $\left\{x_{k}\right\}$, and assume that $x_{k_{j}} \rightarrow \hat{x}$. By the same proof as the one above, we have $\hat{x} \in F(T) \cap(A+B)^{-1} 0$. Moreover, it follows from (3.23) that

$$
\langle(I-f) \tilde{x}, \tilde{x}-\hat{x}\rangle \leq 0
$$

Interchanging $\tilde{x}$ and $\hat{x}$, we obtain

$$
\langle(I-f) \hat{x}, \hat{x}-\tilde{x}\rangle \leq 0
$$

Adding up (3.24) and (3.25) yields

$$
\langle(I-f) \tilde{x}-(I-f) \hat{x}, \tilde{x}-\hat{x}\rangle \leq 0 .
$$


Hence,

$$
\|\tilde{x}-\hat{x}\|^{2} \leq\langle f(\tilde{x})-f(\hat{x}), \tilde{x}-\hat{x}\rangle \leq l\|\tilde{x}-\hat{x}\|^{2},
$$

that is, $(1-l)\|\tilde{x}-\hat{x}\|^{2} \leq 0$. Since $l \in(0,1)$, we have $\tilde{x}=\hat{x}$. Therefore, we conclude that $x_{t} \rightarrow \tilde{x}$ as $t \rightarrow 0$.

Note that $P_{F(T) \cap(A+B)^{-1} 0}$ is well defined by Lemma 2.3 and Remark 2.1. The variational inequality (3.2) can be rewritten as

$$
\langle(2 I-f) \tilde{x}-\tilde{x}, p-\tilde{x}\rangle \geq 0, \quad \forall p \in F(T) \cap(A+B)^{-1} 0 .
$$

By (2.4), this is equivalent to the fixed point equation

$$
\tilde{x}=P_{F(T) \cap(A+B)^{-1} 0}(2 I-f) \tilde{x} .
$$

This completes the proof.

From Theorem 3.1, we can deduce the following result.

Corollary 3.1 Suppose that $F(T) \cap(A+B)^{-1} 0 \neq \emptyset$. Then the net $\left\{x_{t}\right\}$ defined by the implicit method (3.3) converges strongly, as $t \rightarrow 0$, to $\tilde{x}$, which solves the following minimum norm problem: find $\tilde{x} \in F(T) \cap(A+B)^{-1} 0$ such that

$$
\|\tilde{x}\|=\min _{x \in F(T) \cap(A+B)^{-1} 0}\|x\|
$$

Proof From (3.22) with $f \equiv 0$ and $l=0$, we have

$$
\|\tilde{x}-p\|^{2} \leq\langle p, p-\tilde{x}\rangle, \quad \forall p \in F(T) \cap(A+B)^{-1} 0 .
$$

Equivalently,

$$
\langle\tilde{x}, p-\tilde{x}\rangle \geq 0, \quad \forall p \in F(T) \cap(A+B)^{-1} 0 .
$$

This obviously implies that

$$
\|\tilde{x}\|^{2} \leq\langle p, \tilde{x}\rangle \leq\|p\|\|\tilde{x}\|, \quad \forall p \in F(T) \cap(A+B)^{-1} 0 .
$$

It turns out that $\|\tilde{x}\| \leq\|p\|$ for all $p \in F(T) \cap(A+B)^{-1} 0$. Therefore, $\tilde{x}$ is the minimum-norm point of $F(T) \cap(A+B)^{-1} 0$.

\subsection{Strong convergence of the explicit algorithm}

Now, using Theorem 3.1, we establish the strong convergence of an explicit iterative scheme for finding a solution of the variational inequality (3.2), where the constraint set is the common set of the fixed point set $F(T)$ of the $k$-strictly pseudocontractive mapping $T$ and the solution set $(A+B)^{-1} 0$ of the MIP (1.1). 
Theorem 3.2 Suppose that $F(T) \cap(A+B)^{-1} 0 \neq \emptyset$. Let $\left\{\alpha_{n}\right\},\left\{\beta_{n}\right\} \subset(0,1)$ and $\left\{\lambda_{n}\right\} \subset(0,2 \alpha)$ satisfy the following conditions:

(C1) $\lim _{n \rightarrow \infty} \alpha_{n}=0$;

(C2) $\sum_{n=0}^{\infty} \alpha_{n}=\infty$;

(C3) $0<c \leq \beta_{n} \leq d<1$;

(C4) $0<a \leq \lambda_{n} \leq b<2 \alpha$ and $\lim _{n \rightarrow \infty}\left(\lambda_{n}-\lambda_{n+1}\right)=0$.

Let the sequence $\left\{x_{n}\right\}$ be generated iteratively by (3.4):

$$
x_{n+1}=\alpha_{n} f\left(x_{n}\right)+\beta_{n} x_{n}+\left(1-\alpha_{n}-\beta_{n}\right) S J_{\lambda_{n}}\left(x_{n}-\lambda_{n} A x_{n}\right), \quad \forall n \geq 0,
$$

where $x_{0} \in C$ is an arbitrary initial guess. Then the sequence $\left\{x_{n}\right\}$ converges strongly to a point $\tilde{x}$ in $F(T) \cap(A+B)^{-1} 0$, which is the unique solution of the variational inequality (3.2).

Proof First, from condition (C1), without loss of generality, we assume that $\frac{2(1-l) \alpha_{n}}{1-\alpha_{n} l}<1$, and we note that $F(T)=F(S)$. From now, we put $y_{n}=J_{\lambda_{n}}\left(x_{n}-\lambda_{n} A x_{n}\right)$.

We divide the proof several steps as follows.

Step 1. We show that $\left\|x_{n}-p\right\| \leq \max \left\{\left\|x_{0}-p\right\|, \frac{\|f(p)-p\|}{1-l}\right\}$ for all $n \geq 0$ and all $p \in F(T) \cap$ $(A+B)^{-1} 0\left(=F(S) \cap(A+B)^{-1} 0\right)$. Indeed, let $p \in F(T) \cap(A+B)^{-1} 0$. From $p=J_{\lambda_{n}}\left(p-\lambda_{n} A p\right)=$ $S J_{\lambda_{n}}\left(p-\lambda_{n} A p\right), S p=p$ and Lemma 2.5, we get

$$
\begin{aligned}
\left\|y_{n}-p\right\|^{2} & =\left\|J_{\lambda_{n}}\left(x_{n}-\lambda_{n} A x_{n}\right)-J_{\lambda_{n}}(p-r A p)\right\|^{2} \\
& \leq\left\|\left(x_{n}-\lambda_{n} A x_{n}\right)-\left(p-\lambda_{n} A p\right)\right\|^{2} \\
& =\left\|\left(x_{n}-p\right)-\lambda_{n}\left(A x_{n}-A p\right)\right\|^{2} \\
& =\left\|x_{n}-p\right\|^{2}-2 \lambda_{n}\left\langle x_{n}-p, A x_{n}-A p\right\rangle+\lambda_{n}^{2}\left\|A x_{n}-A p\right\|^{2} \\
& \leq\left\|x_{n}-p\right\|^{2}-2 \lambda_{n} \alpha\left\|A x_{n}-A p\right\|^{2}+\lambda_{n}^{2}\left\|A x_{n}-A p\right\|^{2} \\
& =\left\|x_{n}-p\right\|^{2}+\lambda_{n}\left(\lambda_{n}-2 \alpha\right)\left\|A x_{n}-A p\right\|^{2} \\
& \leq\left\|x_{n}-p\right\|^{2} .
\end{aligned}
$$

Using (3.27), we have

$$
\begin{aligned}
\left\|x_{n+1}-p\right\|= & \left\|\alpha_{n} f\left(x_{n}\right)+\beta_{n} x_{n}+\left(1-\alpha_{n}-\beta_{n}\right) S J_{\lambda_{n}}\left(x_{n}-\lambda_{n} A x_{n}\right)-p\right\| \\
= & \left\|\alpha_{n}\left(f\left(x_{n}\right)-p\right)+\beta_{n}\left(x_{n}-p\right)+\left(1-\alpha_{n}-\beta_{n}\right)\left(S y_{n}-S p\right)\right\| \\
\leq & \alpha_{n}\left\|f\left(x_{n}\right)-f(p)\right\|+\alpha_{n}\|f(p)-p\|+\beta_{n}\left\|x_{n}-p\right\| \\
& +\left(1-\alpha_{n}-\beta_{n}\right)\left\|y_{n}-p\right\| \\
\leq & \alpha_{n} l\left\|x_{n}-p\right\|+\alpha_{n}\|f(p)-p\|+\beta_{n}\left\|x_{n}-p\right\|+\left(1-\alpha_{n}-\beta_{n}\right)\left\|x_{n}-p\right\| \\
= & \left(1-(1-l) \alpha_{n}\right)\left\|x_{n}-p\right\|+(1-l) \alpha_{n} \frac{\|f(p)-p\|}{1-l} .
\end{aligned}
$$

Using an induction, we have

$$
\left\|x_{n}-p\right\| \leq \max \left\{\left\|x_{0}-p\right\|, \frac{\|f(p)-p\|}{1-l}\right\} .
$$

Hence, $\left\{x_{n}\right\}$ is bounded, and so are $\left\{y_{n}\right\},\left\{A x_{n}\right\},\left\{f\left(x_{n}\right)\right\}$ and $\left\{S y_{n}\right\}$. 
Step 2. We show that $\lim _{n \rightarrow \infty}\left\|x_{n+1}-x_{n}\right\|=0$. Put $u_{n}=x_{n}-\lambda_{n} A x_{n}$, and define

$$
x_{n+1}=\beta_{n} x_{n}+\left(1-\beta_{n}\right) z_{n}, \quad \forall n \geq 0 .
$$

Then we have

$$
\begin{aligned}
z_{n+1}- & z_{n} \\
= & \frac{x_{n+2}-\beta_{n+1} x_{n+1}}{1-\beta_{n+1}}-\frac{x_{n+1}-\beta_{n} x_{n}}{1-\beta_{n}} \\
= & \frac{\alpha_{n+1} f\left(x_{n+1}\right)+\beta_{n+1} x_{n+1}+\left(1-\alpha_{n+1}-\beta_{n+1}\right) S J_{\lambda_{n+1}} u_{n+1}-\beta_{n+1} x_{n+1}}{1-\beta_{n+1}} \\
& -\frac{\alpha_{n} f\left(x_{n}\right)+\beta_{n} x_{n}+\left(1-\alpha_{n}-\beta_{n}\right) S J_{\lambda_{n}} u_{n}-\beta_{n} x_{n}}{1-\beta_{n}} \\
= & \frac{\alpha_{n+1} f\left(x_{n+1}\right)+\left(1-\alpha_{n+1}-\beta_{n+1}\right) S J_{\lambda_{n+1}} u_{n+1}}{1-\beta_{n+1}} \\
& -\frac{\alpha_{n} f\left(x_{n}\right)+\left(1-\alpha_{n}-\beta_{n}\right) S J_{\lambda_{n}} u_{n}}{1-\beta_{n}} \\
= & \frac{\alpha_{n+1}}{1-\beta_{n+1}} f\left(x_{n+1}\right)-\frac{\alpha_{n}}{1-\beta_{n}} f\left(x_{n}\right)+S J_{\lambda_{n+1}} u_{n+1}-S J_{\lambda_{n}} u_{n} \\
& -\frac{\alpha_{n+1}}{1-\beta_{n+1}} S J_{\lambda_{n+1}} u_{n+1}+\frac{\alpha_{n}}{1-\beta_{n}} S J_{\lambda_{n}} u_{n} \\
= & \frac{\alpha_{n+1}}{1-\beta_{n+1}}\left(f\left(x_{n+1}\right)-S J_{\lambda_{n+1}} u_{n+1}\right)+\frac{\alpha_{n}}{1-\beta_{n}}\left(S J_{\lambda_{n}} u_{n}-f\left(x_{n}\right)\right) \\
& +S J_{\lambda_{n+1}} u_{n+1}-S J_{\lambda_{n}} u_{n} .
\end{aligned}
$$

Since $I-\lambda_{n+1} A$ is nonexpansive for $\lambda_{n+1} \in(0,2 \alpha)$ (by Lemma 2.5), we have

$$
\left\|\left(I-\lambda_{n+1} A\right) x_{n+1}-\left(I-\lambda_{n+1} A\right) x_{n}\right\| \leq\left\|x_{n+1}-x_{n}\right\| .
$$

By the resolvent identity (2.2) and (3.30), we get

$$
\begin{aligned}
& \left\|J_{\lambda_{n+1}} u_{n+1}-J_{\lambda_{n}} u_{n}\right\| \\
& =\left\|J_{\lambda_{n}}\left(\frac{\lambda_{n}}{\lambda_{n+1}} u_{n+1}+\left(1-\frac{\lambda_{n}}{\lambda_{n+1}}\right) J_{\lambda_{n+1}} u_{n+1}\right)-J_{\lambda_{n}} u_{n}\right\| \\
& \leq\left\|\frac{\lambda_{n}}{\lambda_{n+1}} u_{n+1}+\left(1-\frac{\lambda_{n}}{\lambda_{n+1}}\right) J_{\lambda_{n+1}} u_{n+1}-u_{n}\right\| \\
& \leq \frac{\lambda_{n}}{\lambda_{n+1}}\left\|u_{n+1}-u_{n}\right\|+\left|1-\frac{\lambda_{n}}{\lambda_{n+1}}\right|\left\|J_{\lambda_{n+1}} u_{n+1}-u_{n}\right\| \\
& \leq\left\|u_{n+1}-u_{n}\right\|+\left|1-\frac{\lambda_{n}}{\lambda_{n+1}}\right|\left(\left\|u_{n+1}-u_{n}\right\|+\left\|J_{\lambda_{n+1}} u_{n+1}-u_{n}\right\|\right) \\
& \leq\left\|\left(x_{n+1}-\lambda_{n+1} A x_{n+1}\right)-\left(x_{n}-\lambda_{n} A x_{n}\right)\right\| \\
& \quad+\left|\frac{\lambda_{n+1}-\lambda_{n}}{a}\right|\left(\left\|u_{n+1}-u_{n}\right\|+\left\|J_{\lambda_{n+1}} u_{n+1}-u_{n}\right\|\right) \\
& \leq\left\|\left(I-\lambda_{n+1} A\right) x_{n+1}-\left(I-\lambda_{n+1} A\right) x_{n}\right\|+\left|\lambda_{n+1}-\lambda_{n}\right|\left\|A x_{n}\right\|
\end{aligned}
$$




$$
\begin{aligned}
& +\left|\lambda_{n+1}-\lambda_{n}\right| \frac{1}{a}\left(\left\|u_{n+1}-u_{n}\right\|+\left\|J_{\lambda_{n+1}} u_{n+1}-u_{n}\right\|\right) \\
\leq & \left\|x_{n+1}-x_{n}\right\|+\left|\lambda_{n+1}-\lambda_{n}\right| M_{1},
\end{aligned}
$$

where $M_{1}>0$ is an appropriate constant. Hence, from (3.29) and (3.31), we obtain

$$
\begin{aligned}
&\left\|z_{n+1}-z_{n}\right\| \\
& \leq \frac{\alpha_{n+1}}{1-\beta_{n+1}}\left(\left\|f\left(x_{n+1}\right)\right\|+\left\|S J_{\lambda_{n+1}} u_{n+1}\right\|\right)+\frac{\alpha_{n}}{1-\beta_{n}}\left(\left\|S J_{\lambda_{n}} u_{n}\right\|+\left\|f\left(x_{n}\right)\right\|\right) \\
& \quad+\left\|S J_{\lambda_{n+1}} u_{n+1}-S J_{\lambda_{n}} u_{n}\right\| \\
& \leq \frac{\alpha_{n+1}}{1-\beta_{n+1}}\left(\left\|f\left(x_{n+1}\right)\right\|+\left\|S J_{\lambda_{n+1}} u_{n+1}\right\|\right)+\frac{\alpha_{n}}{1-\beta_{n}}\left(\left\|S J_{\lambda_{n}} u_{n}\right\|+\left\|f\left(x_{n}\right)\right\|\right) \\
& \quad+\left\|J_{\lambda_{n+1}} u_{n+1}-J_{\lambda_{n}} u_{n}\right\| \\
& \leq \frac{\alpha_{n+1}}{1-\beta_{n+1}}\left(\left\|f\left(x_{n+1}\right)\right\|+\left\|S J_{\lambda_{n+1}} u_{n+1}\right\|\right)+\frac{\alpha_{n}}{1-\beta_{n}}\left(\left\|S J_{\lambda_{n}} u_{n}\right\|+\left\|f\left(x_{n}\right)\right\|\right) \\
&+\left\|x_{n+1}-x_{n}\right\|+\left|\lambda_{n+1}-\lambda_{n}\right| M_{1} .
\end{aligned}
$$

It follows from conditions $(\mathrm{C} 1)$ and $(\mathrm{C} 4)$ that

$$
\limsup _{n \rightarrow \infty}\left(\left\|z_{n+1}-z_{n}\right\|-\left\|x_{n+1}-x_{n}\right\|\right) \leq 0 .
$$

Thus, by Lemma 2.8, we have

$$
\lim _{n \rightarrow \infty}\left\|z_{n}-x_{n}\right\|=0
$$

Consequently, we obtain

$$
\lim _{n \rightarrow \infty}\left\|x_{n+1}-x_{n}\right\|=\lim _{n \rightarrow \infty}\left(1-\beta_{n}\right)\left\|z_{n}-x_{n}\right\|=0
$$

Step 3. We show that $\lim _{n \rightarrow \infty}\left\|A x_{n}-A p\right\|=0$ for $p \in F(T) \cap(A+B)^{-1}$ 0. From (3.4), (3.27) and Lemma 2.2, we have

$$
\begin{aligned}
&\left\|x_{n+1}-p\right\|^{2} \\
&=\left\|\alpha_{n} f\left(x_{n}\right)+\beta_{n} x_{n}+\left(1-\alpha_{n}-\beta_{n}\right) S J_{\lambda_{n}}\left(x_{n}-\lambda_{n} A x_{n}\right)-p\right\|^{2} \\
&=\left\|\alpha_{n}\left(f\left(x_{n}\right)-p\right)+\beta_{n}\left(x_{n}-p\right)+\left(1-\alpha_{n}-\beta_{n}\right)\left(S y_{n}-p\right)\right\|^{2} \\
&= \alpha_{n}\left\|f\left(x_{n}\right)-p\right\|^{2}+\beta_{n}\left\|x_{n}-p\right\|^{2}+\left(1-\alpha_{n}-\beta_{n}\right)\left\|S y_{n}-p\right\|^{2} \\
&-\alpha_{n} \beta_{n}\left\|f\left(x_{n}\right)-x_{n}\right\|^{2}-\beta_{n}\left(1-\alpha_{n}-\beta_{n}\right)\left\|x_{n}-S y_{n}\right\|^{2} \\
&-\alpha_{n}\left(1-\alpha_{n}-\beta_{n}\right)\left\|S y_{n}-f\left(x_{n}\right)\right\|^{2} \\
& \leq \alpha_{n}\left(\left\|f\left(x_{n}\right)-f(p)\right\|+\|f(p)-p\|\right)^{2}+\beta_{n}\left\|x_{n}-p\right\|^{2}+\left(1-\alpha_{n}-\beta_{n}\right)\left\|y_{n}-p\right\|^{2} \\
& \leq \alpha_{n}\left(l^{2}\left\|x_{n}-p\right\|^{2}+2 l\left\|x_{n}-p\right\|\|f(p)-p\|+\|f(p)-p\|^{2}\right)+\beta_{n}\left\|x_{n}-p\right\|^{2} \\
&+\left(1-\alpha_{n}-\beta_{n}\right)\left\|x_{n}-p\right\|^{2}+\left(1-\alpha_{n}-\beta_{n}\right) \lambda_{n}\left(\lambda_{n}-2 \alpha\right)\left\|A x_{n}-A p\right\|^{2}
\end{aligned}
$$




$$
\begin{aligned}
\leq & \left(1-(1-l) \alpha_{n}\right)\left\|x_{n}-p\right\|^{2}+\left(1-\alpha_{n}-\beta_{n}\right) \lambda_{n}\left(\lambda_{n}-2 \alpha\right)\left\|A x_{n}-A p\right\|^{2} \\
& +\alpha_{n}\left(2 l\left\|x_{n}-p\right\|\|f(p)-p\|+\|f(p)-p\|^{2}\right) \\
\leq & \left\|x_{n}-p\right\|^{2}+\left(1-\alpha_{n}-\beta_{n}\right) \lambda_{n}\left(\lambda_{n}-2 \alpha\right)\left\|A x_{n}-A p\right\|^{2}+\alpha_{n} M_{2},
\end{aligned}
$$

where $M_{2}>0$ is an appropriate constant. From (3.34) and conditions (C3) and (C4), we deduce that

$$
\begin{aligned}
\left(1-\alpha_{n}-d\right) a(2 \alpha-b)\left\|A x_{n}-A p\right\|^{2} & \leq\left(1-\alpha_{n}-\beta_{n}\right) \lambda_{n}\left(2 \alpha-\lambda_{n}\right)\left\|A x_{n}-A p\right\|^{2} \\
& \leq\left\|x_{n}-x_{n+1}\right\|\left(\left\|x_{n}-p\right\|+\left\|x_{n+1}-p\right\|\right)+\alpha_{n} M_{2} .
\end{aligned}
$$

Since $\alpha_{n} \rightarrow 0$ (by condition (C1)) and $\left\|x_{n+1}-x_{n}\right\| \rightarrow 0$ (by Step 2), we conclude that

$$
\lim _{n \rightarrow \infty}\left\|A x_{n}-A p\right\|=0
$$

Step 4. We show that $\lim _{n \rightarrow \infty}\left\|x_{n}-y_{n}\right\|=0$. First, from (2.1) and (2.3), we get for $p \in$ $F(T) \cap(A+B)^{-1} 0$,

$$
\begin{aligned}
\left\|y_{n}-p\right\|^{2}= & \left\|J_{\lambda_{n}}\left(x_{n}-\lambda_{n} A x_{n}\right)-p\right\|^{2} \\
= & \left\|J_{\lambda_{n}}\left(x_{n}-\lambda_{n} A x_{n}\right)-J_{\lambda_{n}}\left(p-\lambda_{n} A p\right)\right\|^{2} \\
\leq & \left\langle\left(x_{n}-\lambda_{n} A x_{n}\right)-\left(p-\lambda_{n} A p\right), y_{n}-p\right\rangle \\
= & \frac{1}{2}\left(\left\|\left(x_{n}-\lambda_{n} A x_{n}\right)-\left(p-\lambda_{n} A p\right)\right\|^{2}+\left\|y_{n}-p\right\|^{2}\right. \\
& \left.-\left\|\left(x_{n}-\lambda_{n} A x_{n}\right)-\left(p-\lambda_{n} A p\right)-\left(y_{n}-p\right)\right\|^{2}\right) \\
\leq & \frac{1}{2}\left(\left\|x_{n}-p\right\|^{2}+\left\|y_{n}-p\right\|^{2}-\left\|x_{n}-y_{n}-\lambda_{n}\left(A x_{n}-A p\right)\right\|^{2}\right) \\
= & \frac{1}{2}\left(\left\|x_{n}-p\right\|^{2}+\left\|y_{n}-p\right\|^{2}-\left\|x_{n}-y_{n}\right\|^{2}\right. \\
& \left.+2 \lambda_{n}\left\langle x_{n}-y_{n}, A x_{n}-A p\right\rangle-\lambda_{n}^{2}\left\|A x_{n}-A p\right\|^{2}\right) .
\end{aligned}
$$

So, we have

$$
\begin{aligned}
\left\|y_{n}-p\right\|^{2} \leq & \left\|x_{n}-p\right\|^{2}-\left\|x_{n}-y_{n}\right\|^{2}+2 \lambda_{n}\left\langle x_{n}-y_{n}, A x_{n}-A p\right\rangle \\
& -\lambda_{n}^{2}\left\|A x_{n}-A p\right\|^{2} \\
\leq & \left\|x_{n}-p\right\|^{2}-\left\|x_{n}-y_{n}\right\|^{2}+2 \lambda_{n}\left\langle x_{n}-y_{n}, A x_{n}-A p\right\rangle .
\end{aligned}
$$

Using (3.34) and (3.35), we obtain

$$
\begin{aligned}
& \left\|x_{n+1}-p\right\|^{2} \\
& \quad \leq \alpha_{n}\left(l^{2}\left\|x_{n}-p\right\|^{2}+2 l\left\|x_{n}-p\right\|\|f(p)-p\|+\|f(p)-p\|^{2}\right)+\beta_{n}\left\|x_{n}-p\right\|^{2} \\
& \quad+\left(1-\alpha_{n}-\beta_{n}\right)\left\|y_{n}-p\right\|^{2} \\
& \leq \alpha_{n} l\left\|x_{n}-p\right\|^{2}+\alpha_{n} M_{2}+\beta_{n}\left\|x_{n}-p\right\|^{2}
\end{aligned}
$$




$$
\begin{aligned}
& +\left(1-\alpha_{n}-\beta_{n}\right)\left(\left\|x_{n}-p\right\|^{2}-\left\|x_{n}-y_{n}\right\|^{2}+2 \lambda_{n}\left\langle x_{n}-y_{n}, A x_{n}-A p\right\rangle\right) \\
= & \left(1-(1-l) \alpha_{n}\right)\left\|x_{n}-p\right\|^{2}-\left(1-\alpha_{n}-\beta_{n}\right)\left\|x_{n}-y_{n}\right\|^{2} \\
& +2 \lambda_{n}\left\langle x_{n}-y_{n}, A x_{n}-A p\right\rangle+\alpha_{n} M_{2} \\
\leq & \left\|x_{n}-p\right\|^{2}-\left(1-\alpha_{n}-\beta_{n}\right)\left\|x_{n}-y_{n}\right\|^{2}+2 b M_{3}\left\|A x_{n}-A p\right\|+\alpha_{n} M_{2},
\end{aligned}
$$

where $M_{2}, M_{3}>0$ are appropriate constants. This implies that

$$
\begin{aligned}
& \left(1-\alpha_{n}-d\right)\left\|x_{n}-y_{n}\right\|^{2} \\
& \quad \leq\left(1-\alpha_{n}-\beta_{n}\right)\left\|x_{n}-y_{n}\right\|^{2} \\
& \quad \leq\left\|x_{n}-x_{n+1}\right\|\left(\left\|x_{n+1}-p\right\|+\left\|x_{n}-p\right\|\right)+2 b M_{3}\left\|A x_{n}-A p\right\|+\alpha_{n} M_{2} .
\end{aligned}
$$

Thus, from condition (C1), Step 2 and Step 3, we deduce that

$$
\lim _{n \rightarrow \infty}\left\|x_{n}-y_{n}\right\|=0 .
$$

Step 5. We show that $\lim _{n \rightarrow \infty}\left\|S x_{n}-x_{n}\right\|=0$. First, by (3.4), we have

$$
\begin{aligned}
\left\|S y_{n}-x_{n}\right\| & \leq\left\|S y_{n}-x_{n+1}\right\|+\left\|x_{n+1}-x_{n}\right\| \\
& =\left\|S y_{n}-\left(\alpha_{n} f\left(x_{n}\right)+\beta_{n} x_{n}+\left(1-\alpha_{n}-\beta_{n}\right) S y_{n}\right)\right\|+\left\|x_{n+1}-x_{n}\right\| \\
& \leq \alpha_{n}\left\|S y_{n}-f\left(x_{n}\right)\right\|+\beta_{n}\left\|x_{n}-S y_{n}\right\|+\left\|x_{n+1}-x_{n}\right\|,
\end{aligned}
$$

and so,

$$
\left\|S y_{n}-x_{n}\right\| \leq \frac{1}{1-\beta_{n}}\left(\alpha_{n}\left\|S y_{n}-f\left(x_{n}\right)\right\|+\left\|x_{n+1}-x_{n}\right\|\right) .
$$

By conditions (C1) and (C3) and Step 2, we obtain

$$
\lim _{n \rightarrow \infty}\left\|S y_{n}-x_{n}\right\|=0
$$

This together with Step 4 yields that

$$
\begin{aligned}
\left\|S x_{n}-x_{n}\right\| & \leq\left\|S x_{n}-S y_{n}\right\|+\left\|S y_{n}-x_{n}\right\| \\
& \leq\left\|x_{n}-y_{n}\right\|+\left\|S y_{n}-x_{n}\right\| \rightarrow 0 \quad \text { as } n \rightarrow \infty .
\end{aligned}
$$

Step 6. We show that

$$
\limsup _{n \rightarrow \infty}\left\langle f(\tilde{x})-\tilde{x}, x_{n}-\tilde{x}\right\rangle \leq 0,
$$

where $\tilde{x}=\lim _{t \rightarrow 0} x_{t}$ with $x_{t}$ being defined by (3.1). We note that from Theorem 3.1, $\tilde{x} \in$ Fix $(T) \cap(A+B)^{-1} 0$, and $\tilde{x}$ is the unique solution of the variational inequality (3.2). To show this, we can choose a subsequence $\left\{x_{n_{i}}\right\}$ of $\left\{x_{n}\right\}$ such that

$$
\lim _{i \rightarrow \infty}\left\langle f(\tilde{x})-\tilde{x}, x_{n_{i}}-\tilde{x}\right\rangle=\limsup _{n \rightarrow \infty}\left\langle f(\tilde{x})-\tilde{x}, x_{n}-\tilde{x}\right\rangle .
$$


Since $\left\{x_{n_{i}}\right\}$ is bounded, there exists a subsequence $\left\{x_{n_{i j}}\right\}$ of $\left\{x_{n_{i}}\right\}$, which converges weakly to $w$. Without loss of generality, we can assume that $x_{n_{i}} \rightarrow w$. By the same argument as in the proof of Theorem 3.1 together with Step 5, we have $w \in F(T) \cap(A+B)^{-1} 0$. Since $\tilde{x}=$ $P_{F(T) \cap(A+B)^{-1} 0}(2 I-f) \tilde{x}$ is the unique solution of the variational inequality (3.2), we deduce that

$$
\begin{aligned}
\limsup _{n \rightarrow \infty}\left\langle f(\tilde{x})-\tilde{x}, x_{n}-\tilde{x}\right\rangle & =\lim _{i \rightarrow \infty}\left\langle f(\tilde{x})-\tilde{x}, x_{n_{i}}-\tilde{x}\right\rangle \\
& =\langle f(\tilde{x})-\tilde{x}, w-\tilde{x}\rangle \leq 0 .
\end{aligned}
$$

Step 7. We show that $\lim _{n \rightarrow \infty}\left\|x_{n}-\tilde{x}\right\|=0$, where $\tilde{x}=\lim _{t \rightarrow 0} x_{t}$ with $x_{t}$ being defined by (3.1), and $\tilde{x}$ is the unique solution of the variational inequality (3.2). Indeed, from (3.4), we note that

$$
\begin{aligned}
x_{n+1}-\tilde{x} & =\alpha_{n} f\left(x_{n}\right)+\beta_{n} x_{n}+\left(1-\alpha_{n}-\beta_{n}\right) S J_{\lambda_{n}}\left(x_{n}-\lambda_{n} A x_{n}\right)-\tilde{x} \\
& =\alpha_{n}\left(f\left(x_{n}\right)-\tilde{x}\right)+\beta_{n}\left(x_{n}-\tilde{x}\right)+\left(1-\alpha_{n}-\beta_{n}\right)\left(S J_{\lambda_{n}} y_{n}-\tilde{x}\right) .
\end{aligned}
$$

Applying Lemma 2.1, we have

$$
\begin{aligned}
\left\|x_{n+1}-\tilde{x}\right\|^{2} \leq & \left\|\beta_{n}\left(x_{n}-\tilde{x}\right)+\left(1-\alpha_{n}-\beta_{n}\right)\left(S J_{\lambda_{n}} y_{n}-\tilde{x}\right)\right\|^{2} \\
& +2 \alpha_{n}\left(f\left(x_{n}\right)-f(\tilde{x}), x_{n+1}-\tilde{x}\right)+2 \alpha_{n}\left\langle f(\tilde{x})-\tilde{x}, x_{n+1}-\tilde{x}\right\rangle \\
\leq & \left(\beta_{n}\left\|x_{n}-\tilde{x}\right\|+\left(1-\alpha_{n}-\beta_{n}\right)\left\|y_{n}-\tilde{x}\right\|\right)^{2} \\
& +2 \alpha_{n} l\left\|x_{n}-\tilde{x}\right\|\left\|x_{n+1}-\tilde{x}\right\|+2 \alpha_{n}\left\langle f(\tilde{x})-\tilde{x}, x_{n+1}-\tilde{x}\right\rangle \\
\leq & \left(1-\alpha_{n}\right)^{2}\left\|x_{n}-\tilde{x}\right\|^{2}+\alpha_{n} l\left(\left\|x_{n}-\tilde{x}\right\|^{2}+\left\|x_{n+1}-\tilde{x}\right\|^{2}\right) \\
& +2 \alpha_{n}\left(f(\tilde{x})-\tilde{x}, x_{n+1}-\tilde{x}\right) .
\end{aligned}
$$

This implies that

$$
\begin{aligned}
\left\|x_{n+1}-\tilde{x}\right\|^{2} \leq & \frac{1-(2-l) \alpha_{n}+\alpha_{n}^{2}}{1-\alpha_{n} l}\left\|x_{n}-\tilde{x}\right\|^{2}+\frac{2 \alpha_{n}}{1-\alpha_{n} l}\left\langle f(\tilde{x})-\tilde{x}, x_{n+1}-\tilde{x}\right\rangle \\
= & \frac{1-(2-l) \alpha_{n}}{1-\alpha_{n} l}\left\|x_{n}-\tilde{x}\right\|^{2}+\frac{\alpha_{n}^{2}}{1-\alpha_{n} l}\left\|x_{n}-\tilde{x}\right\|^{2} \\
& +\frac{2 \alpha_{n}}{1-\alpha_{n} l}\left\langle f(\tilde{x})-\tilde{x}, x_{n+1}-\tilde{x}\right\rangle \\
= & \left(1-\frac{2(1-l) \alpha_{n}}{1-\alpha_{n} l}\right)\left\|x_{n}-\tilde{x}\right\|^{2}+\frac{\alpha_{n}^{2}}{1-\alpha_{n} l}\left\|x_{n}-\tilde{x}\right\|^{2} \\
& +\frac{2 \alpha_{n}}{1-\alpha_{n} l}\left\langle f(\tilde{x})-\tilde{x}, x_{n+1}-\tilde{x}\right\rangle \\
\leq & \left(1-\frac{2(1-l) \alpha_{n}}{1-\alpha_{n} l}\right)\left\|x_{n}-\tilde{x}\right\|^{2} \\
& +\frac{2(1-l) \alpha_{n}}{1-\alpha_{n} l}\left(\frac{\alpha_{n} M_{4}}{2(1-l)}+\frac{1}{1-l}\left\langle f(\tilde{x})-\tilde{x}, x_{n+1}-\tilde{x}\right\rangle\right) \\
= & \left(1-\xi_{n}\right)\left\|x_{n}-\tilde{x}\right\|^{2}+\xi_{n} \delta_{n},
\end{aligned}
$$


where $M_{4}>0$ is an appropriate constant, $\xi_{n}=\frac{2(1-l) \alpha_{n}}{1-\alpha_{n} l}$ and

$$
\delta_{n}=\frac{\alpha_{n} M_{4}}{2(1-l)}+\frac{1}{1-l}\left\langle f(\tilde{x})-\tilde{x}, x_{n+1}-\tilde{x}\right\rangle .
$$

From conditions $(\mathrm{C} 1)$ and $(\mathrm{C} 2)$ and Step 6 , it is easy to see that $\xi_{n} \rightarrow 0, \sum_{n=0}^{\infty} \xi_{n}=\infty$ and $\lim \sup _{n \rightarrow \infty} \delta_{n} \leq 0$. Hence, by Lemma 2.9, we conclude that $x_{n} \rightarrow \tilde{x}$ as $n \rightarrow \infty$. This completes the proof.

From Theorem 3.2, we deduce immediately the following result.

Corollary 3.2 Suppose that $F(T) \cap(A+B)^{-1} 0 \neq \emptyset$. Let $\left\{\alpha_{n}\right\},\left\{\beta_{n}\right\} \subset(0,1)$ and $\left\{\lambda_{n}\right\} \subset(0,2 \alpha)$ satisfy the following conditions:

(C1) $\lim _{n \rightarrow \infty} \alpha_{n}=0$;

(C2) $\sum_{n=0}^{\infty} \alpha_{n}=\infty$;

(C3) $0<c \leq \beta_{n} \leq d<1$;

(C4) $0<a \leq \lambda_{n} \leq b<2 \alpha$.

Let the sequence $\left\{x_{n}\right\}$ be generated iteratively by (3.5):

$$
x_{n+1}=\beta_{n} x_{n}+\left(1-\alpha_{n}-\beta_{n}\right) S J_{\lambda_{n}}\left(x_{n}-\lambda_{n} A x_{n}\right), \quad \forall n \geq 0
$$

where $x_{0} \in C$ is an arbitrary initial guess. Then the sequence $\left\{x_{n}\right\}$ converges strongly to a point $\tilde{x}$ in $F(T) \cap(A+B)^{-1} 0$, which is the unique solution of the minimum norm problem (3.26).

Proof The variational inequality (3.2) is reduced to the inequality

$$
\langle\tilde{x}, p-\tilde{x}\rangle \geq 0, \quad \forall p \in F(T) \cap(A+B)^{-1} 0 .
$$

This is equivalent to $\|\tilde{x}\|^{2} \leq\langle p, \tilde{x}\rangle \leq\|p\|\|\tilde{x}\|$ for all $p \in F(T) \cap(A+B)^{-1} 0$. It turns out that $\|\tilde{x}\| \leq\|p\|$ for all $p \in F(T) \cap(A+B)^{-1} 0$ and $\tilde{x}$ is the minimum-norm point of $F(T) \cap$ $(A+B)^{-1} 0$.

Remark 3.1 It is worth pointing out that our iterative schemes (3.1) and (3.4) are new ones different from those in the literature. The iterative schemes (3.3) and (3.5) are also new ones different from those in the literature (see $[5,11]$ and the references therein).

\section{Applications}

Let $H$ be a real Hilbert space, and let $g$ be a proper lower semicontinuous convex function of $H$ into $(-\infty, \infty]$. Then the subdifferential $\partial g$ of $g$ is defined as follows:

$$
\partial g(x)=\{z \in H \mid g(x)+\langle z, y-x\rangle \leq g(y), y \in H\}
$$

for all $x \in H$. From Rockafellar [19], we know that $\partial g$ is maximal monotone. Let $C$ be a closed convex subset of $H$, and let $i_{C}$ be the indicator function of $C$, i.e.,

$$
i_{C}(x)= \begin{cases}0, & x \in C, \\ \infty, & x \notin C .\end{cases}
$$


Since $i_{C}$ is a proper lower semicontinuous convex function on $H$, the subdifferential $\partial i_{C}$ of $i_{C}$ is a maximal monotone operator. It is well known that if $B=\partial i_{C}$, then the MIP (1.1) is equivalent to find $u \in C$ such that

$$
\langle A u, v-u\rangle \geq 0, \quad \forall v \in C
$$

This problem is called Hartman-Stampacchia variational inequality (see [20]). The set of solutions of the variational inequality (4.2) is denoted by $\operatorname{VI}(C, A)$.

The following result is proved by Takahashi et al. [11].

Lemma 4.1 [11] Let $C$ be a nonempty closed convex subset of a real Hilbert space $H$, let $P_{C}$ be the metric projection from $H$ onto $C$, let $\partial i_{C}$ be the subdifferential of $i_{C}$, and let $J_{\lambda}$ be the resolvent of $\partial i_{C}$ for $\lambda>0$, where $i_{C}$ is defined by (4.1) and $J_{\lambda}=\left(I+\lambda \partial i_{C}\right)^{-1}$. Then

$$
u=J_{\lambda} x \quad \Longleftrightarrow \quad u=P_{C} x, \quad \forall x \in H, y \in C .
$$

Applying Theorem 3.2, we can obtain a strong convergence theorem for finding a common element of the set of solutions to the variational inequality (4.2) and the set of fixed points of a nonexpansive mapping.

Theorem 4.1 Let $C$ be a nonempty closed convex subset of a real Hilbert space H. Let A be an $\alpha$-inverse strongly monotone mapping of $C$ into $H$, and let $S$ be a nonexpansive mapping of $C$ into itself such that $F(S) \cap \operatorname{VI}(C, A) \neq \emptyset$. Let $\left\{\alpha_{n}\right\},\left\{\beta_{n}\right\} \subset(0,1)$ and $\left\{\lambda_{n}\right\} \subset(0,2 \alpha)$ satisfy the following conditions:

(C1) $\lim _{n \rightarrow \infty} \alpha_{n}=0$;

(C2) $\sum_{n=0}^{\infty} \alpha_{n}=\infty$;

(C3) $0<c \leq \beta_{n} \leq d<1$;

(C4) $0<a \leq \lambda_{n} \leq b<2 \alpha$ and $\lim _{n \rightarrow \infty}\left(\lambda_{n}-\lambda_{n+1}\right)=0$.

Let the sequence $\left\{x_{n}\right\}$ be generated iteratively by

$$
x_{n+1}=\alpha_{n} f\left(x_{n}\right)+\beta_{n} x_{n}+\left(1-\alpha_{n}-\beta_{n}\right) S P_{C}\left(x_{n}-\lambda_{n} A x_{n}\right), \quad \forall n \geq 0,
$$

where $x_{0} \in C$ is an arbitrary initial guess. Then the sequence $\left\{x_{n}\right\}$ converges strongly to a point $\tilde{x}$ in $F(S) \cap \operatorname{VI}(C, A)$.

Proof Put $B=\partial i_{C}$. It is easy to show that $\operatorname{VI}(C, A)=\left(A+\partial i_{C}\right)^{-1} 0$. In fact,

$$
\begin{aligned}
x \in\left(A+\partial i_{C}\right)^{-1} 0 & \Longleftrightarrow 0 \in A x+\partial i_{C} x \\
& \Longleftrightarrow \quad-A x \in \partial i_{C} x \\
& \Longleftrightarrow\langle A x, u-x\rangle \geq 0 \quad(\forall u \in C) \\
& \Longleftrightarrow x \in \mathrm{VI}(C, A) .
\end{aligned}
$$

From Lemma 4.1, we get $J_{\lambda_{n}}=P_{C}$ for all $\lambda_{n}$. Hence, the desired result follows from Theorem 3.2. 
As in [11, 21], we consider the problem for finding a common element of the set of solutions of a mathematical model related to equilibrium problems and the set of fixed points of a nonexpansive mapping in a Hilbert space.

Let $C$ be a nonempty closed convex subset of a Hilbert space $H$, and let us assume that a bifunction $\Theta: C \times C \rightarrow \mathbb{R}$ satisfies the following conditions:

(A1) $\Theta(x, x)=0$ for all $x \in C$;

(A2) $\Theta$ is monotone, that is, $\Theta(x, y)+\Theta(y, x) \leq 0$ for all $x, y \in C$;

(A3) for each $x, y, z \in C$,

$$
\lim _{t \downarrow 0} \Theta(t z+(1-t) x, y) \leq \Theta(x, y) ;
$$

(A4) for each $x \in C, y \mapsto \Theta(x, y)$ is convex and lower semicontinuous.

Then the mathematical model related to the equilibrium problem (with respect to $C$ ) is find $\hat{x} \in C$ such that

$$
\Theta(\hat{x}, y) \geq 0
$$

for all $y \in C$. The set of such solutions $\hat{x}$ is denoted by $\operatorname{EP}(\Theta)$. The following lemma was given in $[22,23]$.

Lemma $4.2[22,23]$ Let $C$ be a nonempty closed convex subset of $H$, and let $\Theta$ be a bifunction of $C \times C$ into $\mathbb{R}$ satisfying (A1)-(A4). Then for any $r>0$ and $x \in H$, there exists $z \in C$ such that

$$
\Theta(z, y)+\frac{1}{r}\langle y-z, z-x\rangle \geq 0, \quad \forall y \in C .
$$

Moreover, if we define $T_{r}: H \rightarrow C$ as follows:

$$
T_{r} x=\left\{z \in C: \Theta(z, y)+\frac{1}{r}\langle y-z, z-x\rangle \geq 0, \forall y \in C\right\}
$$

for all $x \in H$, then the following hold:

(1) $T_{r}$ is single-valued;

(2) $T_{r}$ is firmly nonexpansive, that is, for any $x, y \in H$,

$$
\left\|T_{r} x-T_{r} y\right\|^{2} \leq\left\langle T_{r} x-T_{r} y, x-y\right\rangle
$$

(3) $F\left(T_{r}\right)=\mathrm{EP}(\Theta)$;

(4) $\mathrm{EP}(\Theta)$ is closed and convex.

We call such $T_{r}$ the resolvent of $\Theta$ for $r>0$. The following lemma was given in Takahashi et al. [11].

Lemma 4.3 [11] Let $C$ be a nonempty closed convex subset of a real Hilbert space $H$, and let $\Theta$ be a bifunction of $C \times C$ into $\mathbb{R}$ satisfying (A1)-(A4). Let $A_{\Theta}$ be a multivalued mapping 
of $H$ into itself defined by

$$
A_{\Theta} \mathcal{X}= \begin{cases}\{z \in H: \Theta(x, y) \geq\langle y-x, z\rangle\}, & x \in C, \\ \emptyset, & x \notin C .\end{cases}
$$

Then $\operatorname{EP}(\Theta)=A_{\Theta}^{-1} 0$, and $A_{\Theta}$ is a maximal monotone operator with $\operatorname{dom}\left(A_{\Theta}\right) \subset C$. Moreover, for any $x \in H$ and $r>0$, the resolvent $T_{r}$ of $\Theta$ coincides with the resolvent of $A_{\Theta}$; i.e.,

$$
T_{r} x=\left(I+r A_{\Theta}\right)^{-1} x
$$

Applying Lemma 4.3 and Theorem 3.2, we can obtain the following results.

Theorem 4.2 Let $C$ be a nonempty closed convex subset of a real Hilbert space $H$, and let $\Theta$ be a bifunction of $C \times C$ into $\mathbb{R}$ satisfying (A1)-(A4). Let $A_{\Theta}$ be a maximal monotone operator with $\operatorname{dom}\left(A_{\Theta}\right) \subset C$ defined as in Lemma 4.3, and let $T_{\lambda}$ be the resolvent of $\Theta$ for $\lambda>0$. Let $A$ be an $\alpha$-inverse strongly monotone mapping of $C$ into $H$, and let $S$ be a nonexpansive mapping from $C$ into itself such that $F(S) \cap\left(A+A_{\Theta}\right)^{-1} 0 \neq \emptyset$. Let $\left\{\alpha_{n}\right\},\left\{\beta_{n}\right\} \subset$ $(0,1)$ and $\left\{\lambda_{n}\right\} \subset(0,2 \alpha)$ satisfy the following conditions:

(C1) $\lim _{n \rightarrow \infty} \alpha_{n}=0$;

(C2) $\sum_{n=0}^{\infty} \alpha_{n}=\infty$;

(C3) $0<c \leq \beta_{n} \leq d<1$;

(C4) $0<a \leq \lambda_{n} \leq b<2 \alpha$ and $\lim _{n \rightarrow \infty}\left(\lambda_{n}-\lambda_{n+1}\right)=0$.

Let the sequence $\left\{x_{n}\right\}$ be generated iteratively by

$$
x_{n+1}=\alpha_{n} f\left(x_{n}\right)+\beta_{n} x_{n}+\left(1-\alpha_{n}-\beta_{n}\right) S T_{\lambda_{n}}\left(x_{n}-\lambda_{n} A x_{n}\right), \quad \forall n \geq 0,
$$

where $x_{0} \in C$ is an arbitrary initial guess. Then the sequence $\left\{x_{n}\right\}$ converges strongly to a point $\tilde{x}$ in $F(S) \cap\left(A+A_{\Theta}\right)^{-1} 0$.

Theorem 4.3 Let $C$ be a nonempty closed convex subset of a real Hilbert space $H$, and let $\Theta$ be a bifunction of $C \times C$ into $\mathbb{R}$ satisfying (A1)-(A4). Let $A_{\Theta}$ be a maximal monotone operator with $\operatorname{dom}\left(A_{\Theta}\right) \subset C$ defined as in Lemma 4.3, and let $T_{\lambda}$ be the resolvent of $\Theta$ for $\lambda>0$, and let $S$ be a nonexpansive mapping from $C$ into itself such that $F(S) \cap \operatorname{EP}(\Theta) \neq \emptyset$. Let $\left\{\alpha_{n}\right\},\left\{\beta_{n}\right\} \subset(0,1)$ and $\left\{\lambda_{n}\right\} \subset(0,2 \alpha)$ satisfy the following conditions:

(C1) $\lim _{n \rightarrow \infty} \alpha_{n}=0$;

(C2) $\sum_{n=0}^{\infty} \alpha_{n}=\infty$;

(C3) $0<c \leq \beta_{n} \leq d<1$;

(C4) $0<a \leq \lambda_{n} \leq b<2 \alpha$ and $\lim _{n \rightarrow \infty}\left(\lambda_{n}-\lambda_{n+1}\right)=0$.

Let the sequence $\left\{x_{n}\right\}$ be generated iteratively by

$$
x_{n+1}=\alpha_{n} f\left(x_{n}\right)+\beta_{n} x_{n}+\left(1-\alpha_{n}-\beta_{n}\right) S T_{\lambda_{n}}\left(x_{n}\right), \quad \forall n \geq 0,
$$

where $x_{0} \in C$ is an arbitrary initial guess. Then the sequence $\left\{x_{n}\right\}$ converges strongly to a point $\tilde{x}$ in $F(S) \cap \mathrm{EP}(\Theta)$.

Proof Put $A=0$ in Theorem 4.2. From Lemma 4.3, we also know that $J_{\lambda_{n}}=T_{\lambda_{n}}$ for all $n \geq 0$. Hence, the desired result follows from Theorem 4.2. 
Remark 4.1 (1) As in Corollary 3.2, if we take $f \equiv 0$ in Theorems 4.1, 4.2 and 4.3, then we can obtain the minimum-norm point of $F(S) \cap \mathrm{VI}(C, A), F(S) \cap\left(A+A_{\Theta}\right)^{-1} 0$ and $F(S) \cap$ $\mathrm{EP}(\Theta)$, respectively.

(2) For several iterative schemes for zeros of monotone operators, variational inequality problems, generalized equilibrium problems, convex minimization problems, and fixed point problems, we can also refer to [24-29] and the references therein. By combining our methods in this paper and methods in [24-29], we will consider new iterative schemes for the above-mentioned problems coupled with the fixed point problems of nonlinear operators.

\section{Competing interests}

The author declares that they have no competing interests.

\section{Acknowledgements}

The author would like to thank the anonymous referees for their careful reading and valuable suggestions, which improved the presentation of this manuscript, and the editor for his valuable comments along with providing some recent related papers. This research was supported by the Basic Science Research Program through the National Research Foundation of Korea (NRF) funded by the Ministry of Education, Science and Technology (2013021600).

Received: 19 June 2013 Accepted: 3 September 2013 Published: 08 Nov 2013

\section{References}

1. Chang, SS: Existence and approximation of solutions for set-valued variational inclusions in Banach spaces. Nonlinear Anal. 47, 583-594 (2001)

2. Demyanov, VF, Stavroulakis, GE, Polyakova, LN, Panagiotopoulos, PD: Quasi-Differentiability and Nonsmooth Modelling in Mechanics, Engineering and Economics, vol. 10. Kluwer Academic, Dordrecht (1996)

3. Lin, L, Wang, SY, Chuang, CS: Existence theorems of systems of variational inclusion problems with applications. J. Glob. Optim. 40, 751-764 (2008)

4. Peng, JW, Wang, Y, Shyu, DS, Yao, JC: Common solutions of an iterative scheme for variational inclusions, equilibrium problems, and fixed point problems. J. Inequal. Appl. 2008, Article ID 720371 (2008). doi:10.1155/2008/720371

5. Zhang, SS, Lee, JHW, Chan, CK: Algorithms of common solutions to quasi variational inclusion and fixed point problems. Appl. Math. Mech. 29, 571-581 (2008)

6. Browder, FE, Petryshn, WV: Construction of fixed points of nonlinear mappings Hilbert space. J. Math. Anal. Appl. 20, 197-228 (1967)

7. Acedo, GL, Xu, HK: Iterative methods for strictly pseudo-contractions in Hilbert space. Nonlinear Anal. 67, 2258-2271 (2007)

8. Jung, JS: Strong convergence of iterative methods for k-strictly pseudo-contractive mappings in Hilbert spaces. Appl. Math. Comput. 215, 3736-3753 (2010)

9. Jung, JS: Some results on a general iterative method for k-strictly pseudo-contractive mappings. Fixed Point Theory Appl. 2011, 24 (2011). doi:10.1186/1687-1812-2011-24

10. Morales, $\mathrm{CH}$, Jung, JS: Convergence of paths for pseudo-contractive mappings in Banach spaces. Proc. Am. Math Soc. 128, 3411-3419 (2000)

11. Takahashi, S, Takahashi, W, Toyoda, M: Strong convergence theorems for maximal monotone operators with nonlinear mappings in Hilbert spaces. J. Optim. Theory Appl. 147, 27-41 (2010)

12. Takahashi, W: Nonlinear Functional Analysis: Fixed Point Theory and Its Applications. Yokohama Publishers, Yokohama (2000)

13. Zhou, $\mathrm{H}$ : Convergence theorems of fixed points for $k$-strict pseudo-contractions in Hilbert spaces. Nonlinear Anal. 69 456-462 (2008)

14. Nadezhkina, N, Takahashi, W: Weak convergence theorem by an extragradient method for nonexpansive mappings and monotone mappings. J. Optim. Theory Appl. 128, 191-201 (2006)

15. Brézis, H: Opérateurs maximaux monotones et semi-groupes de contractions dans les espaces de Hilbert. North-Holland Mathematics Studies, vol. 5.Notas de Matemática (50). North-Holland, Amsterdam (1973)

16. Minty, GJ: On the generalization of a direct method of the calculus of variations. Bull. Am. Math. Soc. 73, 315-321 (1967)

17. Suzuki, T: Strong convergence of Krasnoselskii and Mann's type sequences for one parameter nonexpansive semigroups without Bochner integral. J. Math. Anal. Appl. 305, 227-239 (2005)

18. Xu, HK: Iterative algorithms for nonlinear operators. J. Lond. Math. Soc. 66, 240-256 (2002)

19. Rockafellar, RT: On the maximal monotonicity of subdifferential mappings. Pac. J. Math. 33, 209-216 (1970)

20. Hartman, P, Stampacchia, G: On some non-linear elliptic differential-functional equations. Acta Math. 115, 271-310 (1966)

21. Takahashi, S, Takahashi, W: Strong convergence theorem for a generalized equilibrium problem and a nonexpansive mapping in Hilbert spaces. Nonlinear Anal. 69, 1025-1033 (2008)

22. Blum, E, Oettli, W: From optimization and variational inequalities to equilibrium problems. Math. Stud. 63, 123-145 (1994) 
23. Combettes, PI, Hirstoaga, SA: Equilibrium programming in Hilbert spaces. J. Nonlinear Convex Anal. 6, 117-136 (2005)

24. Ceng, LC, Ansari, QH, Khan, AR, Yao, JC: Strong convergence on composite iterative schemes for zeros of $m$-accretive operators in Banach spaces. Nonlinear Anal. 70, 1830-1840 (2009)

25. Ceng, LC, Ansari, QH, Khan, AR, Yao, JC: Viscosity approximation methods for strongly positive and monotone operators. Fixed Point Theory 10, 35-71 (2009)

26. Ceng, LC, Ansari, QH, Yao, JC: Some iterative methods for finding fixed point and for solving constrained convex minimization problems. Nonlinear Anal. 74(16), 5286-5302 (2011)

27. Ceng, LC, Ansari, QH, Yao, JC: Extragradient-projection method for solving constrained convex minimization problems. Numer. Algebra Control Optim. 1(3), 341-359 (2011)

28. Ceng, LC, Ansari, QH, Wong, MM, Yao, JC: Mann type hybrid extragradient method for variational inequalities, variational inclusion and fixed point problems. Fixed Point Theory 13(2), 403-422 (2012)

29. Zeng, LC, Ansari, QH, Shyu, DS, Yao, JC: Strong and weak convergence theorems for common solutions of generalized equilibrium problems and zeros of maximal monotone operators. Fixed Point Theory Appl. 2010, Article ID $590278(2010)$

10.1186/1687-1812-2013-272

Cite this article as: Jung: Iterative algorithms for monotone inclusion problems, fixed point problems and minimization problems. Fixed Point Theory and Applications 2013, 2013:272

\section{Submit your manuscript to a SpringerOpen ${ }^{\ominus}$ journal and benefit from:}

- Convenient online submission

Rigorous peer review

- Immediate publication on acceptance

- Open access: articles freely available online

- High visibility within the field

- Retaining the copyright to your article 